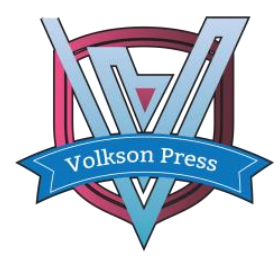

Contents List available at VOLKSON PRESS

Mechanical and Control Engineering (MCE)

DOI : http://doi.org/10.26480/wsmce.01.2017.174.176

\title{
THE INDOOR WIFI LOCALIZATION METHOD BASED ON THE FINGERPRINT LIBRARY NOISE FILTERING
}

\author{
Zou Zi-ming, Li Fu-min, He Wen-bin \\ College of Mechanical and Control Engineering Guilin University of Technology, Guilin,541004, Guangxi,China \\ *Corresponding Author Email: 1945650116@qq.com
}

This is an open access article distributed under the Creative Commons Attribution License, which permits unrestricted use, distribution, and reproduction in any medium, provided the original work is properly cited

\section{ARTICLE DETAILS}

\section{Article History:}

Received 02 october 2017 Accepted 06 october 2017 Available online 11 november 2017

Keywords

indoor positioning, WIFI, RSSI fingerprint matching, Neighborhood mean filtering

\section{ABSTRACT}

Based on the RSSI fingerprint matching positioning technology is widely used in WIFI indoor positioning field, due to the influence of multipath effect, scattering, refraction, staff walking, propagation distance and other aspects, indoor signal propagation is easy to introduce noise into the acquisition signal database, leading to the final positioning result is not too ideal. So, this paper proposes a fingerprint library filtering algorithm based on neighborhood mean filter, introduce the concept of acquisition noise on the basis of traditional algorithm, removes the noise of the original data and reduces the average error of location. The results show the effectiveness of this method, and the improved algorithm makes the positioning accuracy greatly improved.

\section{INTRODUCTION}

Signal propagation in the indoor environment will be blocked by obstacles such as walls, glass and ceilings, so that the transmitted signals will pass through different paths and arrive at the receiving end at different times, causing multipath propagation and non-line of sight propagation phenomena. In addition, the frequent movement of indoor staff, the humidity and temperature of the indoor environment will have a certain effect on the intensity of the signal, which leads to the poor positioning accuracy. At the present stage, WIFI has become the research hotspot of indoor positioning technology because of its advantages such as low erection cost, no additional modules, easy expansion and wide coverage [1].

The technology based on WIFI signal intensity mainly includes two kinds of location method based on distance loss model and location method of location fingerprint [2]. In complex indoor environment, there is a large error in the positioning of RSSI obtained by using the path loss model directly, but location fingerprinting method can effectively avoid such a complex indoor environment, it first establishes a fingerprint database with unknown nodes.

\section{LOCATION ALGORITHM BASED ON FINGERPRINT RECOGNITION}

Fingerprint location system is a location method based on matching idea, the optimal estimation is obtained by matching the signal intensity in the location area with the information in the fingerprint library. This method is generally divided into two stages: offline stage and online stage [3]. The positioning process is shown in Figure 1.

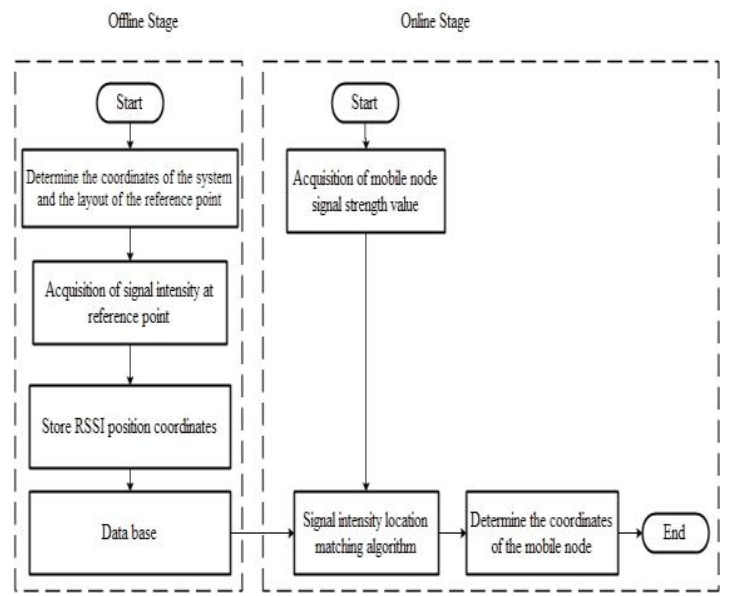

Figure 1: Indoor location flow chart based on fingerprint identification and location algorithm

\section{$2.1 \quad$ Offline acquisition stage}

The main goal of offline stage is to set up a location fingerprint database. First, select a reasonable reference point to ensure that it can provide enough information for accurate location estimation in the positioning stage. Then, by collecting training sequences, RSSI values from different APs are measured at each reference point, together with the corresponding location information of the MAC address and the reference point, an RSSI signal vector is formed and stored in the database, after collecting all the fingerprint points in the area, the database is established.

\section{$2.2 \quad$ Online positioning stage}

At present, RSSI fingerprint positioning algorithms are mainly divided into two types: probabilistic type and k-nearest-neighbor method. Probabilistic algorithms calculate and match all the nodes, so the positioning accuracy is high, but the calculation is large, the calculation process is relatively complex and time-consuming, affect the final positioning efficiency, while k-nearest-neighbor method only need to 
select the best reference point, so the calculation is small, the accuracy is slightly reduced. Taking into account the real-time of the positioning system, this paper uses k-nearest-neighbor method as the basic algorithm to ensure the positioning speed.

The k-nearest-neighbor method is a typical deterministic algorithm, based on the matching method of analogy learning, it is to match the similarity degree between the sampling examples of the positioning stage and the sampling samples in the training stage, the RSSI value in the training phase is called the location fingerprint, using Euclidean distance to describe the similarity between localize fingerprints and position fingerprints, find the coordinates of the $\mathrm{k}$ points with the highest similarity, and determine the coordinates of the unknown location points through the k points [4]. Suppose the RSSI vector collected in the online stage at the time of $\mathrm{t}$ is $f_{r}$, the RSSI vector in the fingerprint database at reference point $R P_{j}$ is $f_{j}$, the distance between two vectors is:

$d_{j}\left(f_{r}, f_{j}\right)=\left\|f_{r}-f_{j}\right\|^{2}$

Where $f_{j}=\left[f_{1, j}, \ldots \ldots f_{L, j}\right]^{T}$ represents the average signal strength vector received from L APs at reference point $R P_{j}$, select $\mathrm{k}$ closest reference points, and estimate the position coordinates of the mobile terminal by averaging [5]:

$(\bar{x}, \bar{y})=\frac{1}{k} \sum_{i=1}^{k}\left(x_{i}, y_{i}\right)$

The selection of $\mathrm{K}$ value is a key factor in k-nearest-neighbor algorithm, which directly affects the complexity of the training phase. In addition, there will be a certain number of noise signals in the selected reference point, which will eventually have adverse effect on the positioning result and reduce the positioning accuracy. However, the Euclidean distance by RSSI value calculated can not remove the interference factor. It needs to perform noise filtering on the data in the fingerprint database before $\mathrm{k}$ nearest-neighbor matching so as to improve the positioning accuracy.

\section{OPTIMIZED MATCHING ALGORITHM}

Based on the k-nearest-neighbor matching algorithm mentioned above, noise filtering is done by using the neighborhood mean filtering method commonly used in image denoising [6-8].

\subsection{Determination of noise points}

In the unobstructed adjacent space, the value of the AP signal should be similar in the fingerprint point, and the similarity is calculated by the formula. When two points are normal points, then the similarity is high, on the contrary, if there is a noise point between two points, the similarity is very small. Construct a neighborhood map of point $A$ from the fingerprint database, and adopt 8-neighborhood system to segment it. The specific algorithm is as follows:

With $A$ as the center of the neighborhood, a total similarity between a point $P_{i}\left(P_{i} \neq A\right)$ and its neighbors are $S_{P_{i}}^{N_{A}}$

$$
S_{P_{i}}^{N_{A}}=\sum_{j \neq i}^{U_{p_{A}}^{N_{A}}} L\left(P_{i}, P_{j}\right), P_{j} \in U_{P_{i}^{N_{A}}}^{N_{1}}
$$

Where, $U_{P_{i}}^{N_{A}}$ represents a set of points adjacent to point $P_{i}$ in a neighborhood centered at $A$

The mean value of the similarity between $P_{i}\left(P_{i} \neq A\right)$ and its adjacent points is $\bar{S}_{P_{i}}^{N_{A}}$ :

$$
\bar{S}_{P_{i}}^{N_{A}}=\frac{S_{P_{i}}^{N_{A}}}{n_{p_{i}}}
$$

In the form, $n_{p_{i}}$ is the number of the $P_{i}\left(P_{i} \neq A\right)$ neighborhood points The average similarity $\bar{S}_{N_{C}^{0}}$ of all the points except for the center point $A$ in domain $N_{A}$ is:

$$
\bar{S}_{N_{C}^{0}}=\frac{1}{8} \sum_{i=1}^{8} \bar{S}_{P_{l}}^{N_{C}}
$$

The similarities between point $A$ and its similarity sum $S_{A}^{N_{A}}$ and mean $\bar{S}_{A}^{N_{A}}$ are:

$$
\begin{aligned}
& S_{A}^{N_{A}}=\sum_{i=1}^{8} L\left(C, P_{i}\right) \\
& \bar{S}_{A}^{N_{A}}=\frac{1}{8} S_{A}^{N_{A}}
\end{aligned}
$$

When $\bar{S}_{A}^{N_{A}}>\bar{S}_{N_{A}^{0}}$, the decision point is the noise point

\section{2 $\quad$ Filtering of noise points}

The signal intensity of the noise point is estimated by the interpolation of the signal intensity of the non-noise point in the neighborhood, and the filtering effect is achieved. The filtering calculation method is as follows:

$$
R_{N}=\frac{1}{n} \sum_{i}^{n} R_{i}
$$

In the formula: $R_{N}$ is the estimated signal strength of the updated noise point, $R_{i}$ is the signal strength measurement of the non-noise point in the neighborhood, and $\mathrm{n}$ is the number of finger points of the non-noise point in the neighborhood.

\subsection{The implementation process of the algorithm}

The fingerprint filtering algorithm based on domain mean filter method is as follows [9-11]:

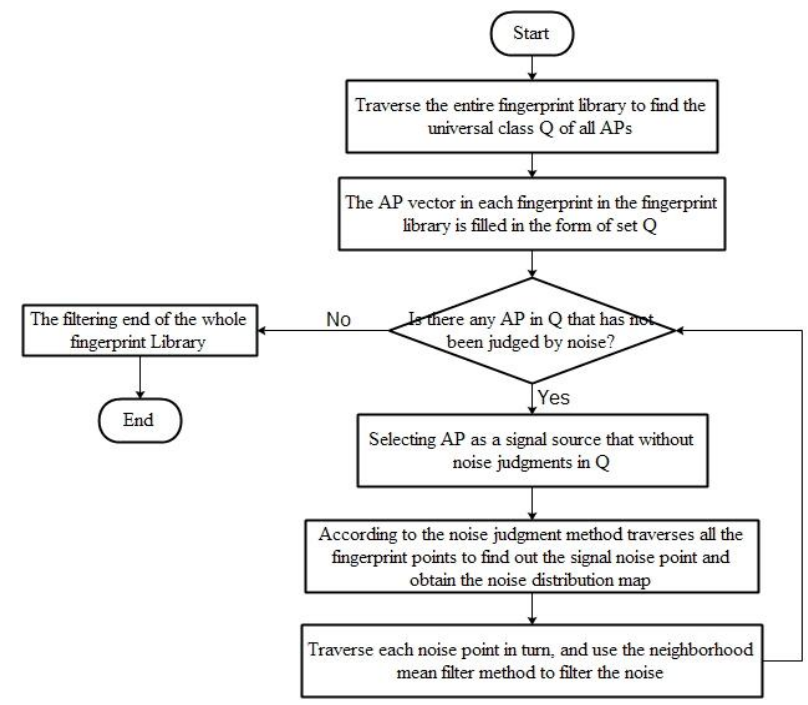

Figure 2: Flow chart of fingerprint library filtering algorithm based on neighborhood mean filtering

\section{EXPERIMENTAL DESIGN AND RESULT ANALYSIS}

\subsection{Experimental environment setting}

The experimental site was selected as the laboratory of the Institute of mechanical and control engineering of Guilin University of Technology. The laboratory area of $18 \mathrm{~m}$ in length and $12 \mathrm{~m}$ in width is the experimental test area. The equipment of desks, chairs and air conditioners in the test area is kept as the same as before, so as to maintain a complex and authentic indoor environment. There are 4 hotspots in WIFI signal. 23 points are selected randomly as experimental points. The triangulation points represent the reference points and the square points represent the randomly selected test points. The layout of the experimental area is shown in Figure 3. 


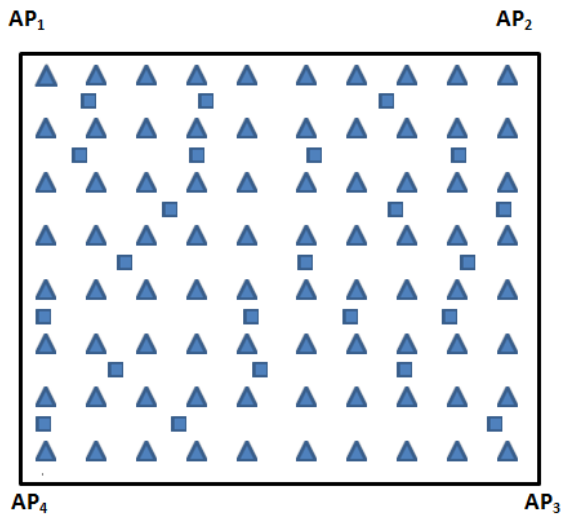

Figure 3: Experimental area layout

In the early stage of data acquisition, the center point of each grid area is selected as a reference point. In order to reduce the system error, 50 sets of data are collected for each sampling point, and the collected data includes the intensity of the wireless signal scanned to each AP, the coordinates of the sampling point, the MAC address of the AP, the mean value of the signal intensity of the sampled data is the final sample value of the sampling point, which are then stored in the database. Finally, the location of the test points for positioning test.

\subsection{Results Analysis}

By comparing the positioning error of the location simulation before and after the noise filtering of the fingerprint library to verify the effect, and the comparison of the positioning results is shown in Figure 4.

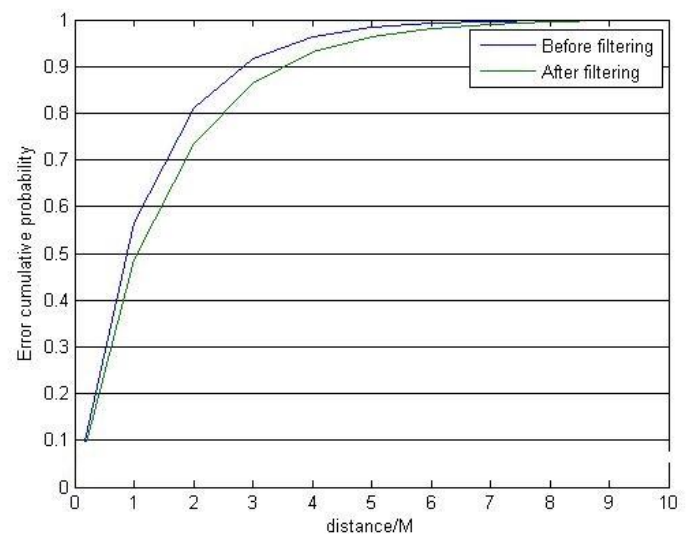

Figure 4: Comparison map of location results

From this diagram, it can be clearly seen that the location effect after fingerprint filtering is obviously better than that before filtering, and the cumulative probability of error decreases with the improved algorithm. The positioning accuracy is higher than that before, and the cumulative probability of error within $2 \mathrm{~m}$ is $73 \%$. Compared with the $82 \%$ of the preoptimization algorithm, there is a significant improvement.

\section{CONCLUSION}

Based on the k-nearest neighbor matching algorithm, this paper uses the neighborhood mean filtering method commonly used in image denoising to filter out the noise, so as to improve the reliability of fingerprint database matching and positioning. The feasibility of this method is verified by experiments. The final positioning accuracy has increased.

\section{REFERENCES}

[1] Xiaoxiao, X., Linbo, X., Li, P. 2015. Design of Indoor Positioning System Based on WIFI Signal Strength Characteristics [J]. Computer Engineering, 40 (1), 87-91.

[2] Jing, X. 2017. Research on the indoor location algorithm based on WIFI [D]. Computer science and technology.

[3] Peiguang, Z. 2014. Research and implementation of indoor positioning system based on Zigbee technology [D]. Hangzhou: Hangzhou Dianzi University, 14-15.

[4] Bahl, P., Padmanabhan, V.N. 2000. RADAR:an in-building RF-based location and tracking system [j]. Institute of Electrical and Electronics Engineers Inc, 2 (1), 775-784

[5] Xiaoliang, Z., Ping, Z., Guanqing, X. 2013. Application research based on an optimized KNN algorithm in indoor positioning [J]. Electronic design engineering, 21 (7), 44-46.

[6] Weiwei, L., Yunhui, Y., Hongwei, S. 2008. An image denoising algorithm based on neighborhood noise evaluation method [J]. University Journal, 29 (7), 1033-1035.

[7] Xiuqin, N. 2012. Improved domain mean filter denoising algorithm [J]. Journal of Changzhi University, 29 (2), 4-8.

[8] Xiufang, W. 2010. The noise of digital image and several methods of denoising [J]. Heilongjiang science and technology information, 12 (031), 53-59.

[9] Brunato, M., Battiti, R. 2005. Statistical learning theory for location fingerprinting in wireless LANs [J]. Computer Networks, 47 (6), 825-848.

[10] Xu, R., Wunsch, D. 2005. Survey of clustering algorithms [J]. Neural Networks, IEEE Transactions on, 16 (3), 645-678.

[11] Zhongliang, D., Yanpei, Y. 2013. Indoor and outdoor wireless positioning and navigation [J]. Beijing University of Posts and Telecommunications, 77-81. 